\title{
Swelling Behavior of Polyacrylamide Hydrogels near Phase Transition
}

\author{
R. R. Janot Pacheco, M. E. S. R. Silva, R. G. Sousa, R. F. S. Freitas \\ Chemical Engineering Department, Engineering School, Federal University of Minas Gerais, Belo Horizonte, \\ Brazil \\ Email: freitas@deq.ufmg.br
}

Received 16 April 2014; revised 18 May 2014; accepted 2 June 2014

Copyright (C) 2014 by authors and Scientific Research Publishing Inc.

This work is licensed under the Creative Commons Attribution International License (CC BY).

http://creativecommons.org/licenses/by/4.0/

(c) (i) Open Access

\begin{abstract}
Hydrogels based on acrylamide (AA) and sodium methacrylate (NMA) as ionic monomer were prepared by solution polymerization using $N, N^{\prime}$-methylenebis(acrylamide) (MBA) or ethylene glycol dimethacrylate (EGMA) as crosslinkers and Ammonium Persulfate (APS) and N,N,N',N'-Tetramethyl-ethylenediamine (TMEDA) as initiators. Swelling behavior was greatly affected by NMA content near phase transition. Increasing ionic monomer concentration compared to total monomer one led to high expansion in water, oscillating around $285 \mathrm{~g} / \mathrm{g}$ for MBA and $325 \mathrm{~g} / \mathrm{g}$ for EGMA crosslinker, above $20 \%$ of NMA. Dynamic Light Scattering experiments were performed and, for both crosslinkers, the dynamic correlation length $(\xi)$ decreased with increasing NMA content, contributing to diminish hydrogels spatial inhomogeneities.
\end{abstract}

\section{Keywords}

Polyacrylamide Hydrogels, Phase Transition, Swelling Behavior, Dynamic Correlation Length

\section{Introduction}

Superabsorbent polymers (SAP) represent a special polymer class with great swelling capacity in water, compared to other absorbent materials [1]. This is possible because they are lightly crosslinked, forming a three-dimensional network structure. Depending on the backbone charges, these materials can be categorized in ionic and non-ionic.

The SAP production for personal care products, such as baby diapers and feminine incontinence products, accounts for about eight percent of its production, but research over the past 30 years has led to new applications in several areas, such as agriculture, pharmaceutics, separation technology, electrical and construction [2].

Several authors have been developing different SAPs based on acrylamide, with different ionic monomers. 
For instance, Mohana Raju et al. have studied hydrogels with calcium, sodium and potassium methacrylate and evaluated their swelling capacity under different conditions [3]-[6]. Karadag et al. have studied hydrogels with sodium acrylate and crotonic acid with different crosslinkers [7]-[9]. More recently they've published new research of hydrogels using sodium methacrylate as ionic monomer and urea as biopotencial sorbent [10]. Berna Yildiz et al. have developed thermoresponsive hydrogels based on isopropylacrylamide and acrylamide [11]. Other authors have studied swelling behavior not only in water but also in saline solutions [12]-[14].

The aim of this study was to develop new hydrogels based on Acrylamide (AAm) and Sodium Methacrylate (NMA) as ionic monomer, using different crosslinkers, find their phase transition and evaluate their swelling behavior.

Non-idealities during gelation necessarily result in hydrogels with a large number of network defects, affecting their physical properties such as swelling, elasticity, transparency, and permeability. One of these defects is the inhomogeneous distribution of the crosslink points along the gel sample, which is known as the spatial gel inhomogeneity [15]. Since the ionization of polymer hydrogels is one of the methods used to suppress these inhomogeneities, dynamic light scattering experiments were also performed.

\section{Experimental}

The experiments carried out in this work involved the hydrogel syntheses, the determination of their amount of swelling at equilibrium and dynamic light scattering measurements.

\subsection{Materials}

Acrylamide (AAm) and Sodium Methacrylate (NMA) were used as monomers, N,N'-methylenebis(acrylamide) (MBA) and ethylene glycol dimethacrylate (EGDMA) were used as crosslinkers and Ammonium Persulfate (APS) and N,N,N',N'-Tetramethyl-ethylenediamine (TMEDA) were used as initiators. All chemicals were supplied by Sigma-Aldrich with exception of APS, which was supplied by Mallinckrodt Chemicals.

\subsection{Hydrogels Syntheses}

Hydrogels were synthesized by free-radical copolymerization of AAm and NMA, using water as solvent. Jerten nomenclature was used to specify the amounts of each reagent in solution. It is composed by three parameters: $\mathrm{X}: \mathrm{Y}: \mathrm{Z}$, where $\mathrm{X}$ is total monomer concentration (w/V \%), $\mathrm{Y}$ is crosslinker concentration per $\mathrm{X}$ ratio (w/w \%) and $\mathrm{Z}$ is ionic monomer concentration per $\mathrm{X}$ ratio (w/w \%).

Different amounts of monomers were weighted in the same beaker, the desired amount of crosslinker was added, followed by $15 \mathrm{ml}$ of water. The mixture was stirred for reagent's dissolution and then $0.05 \mathrm{~g}$ of APS was weighed, dissolved in $160 \mu \mathrm{L}$ of TMEDA $10 \%$ solution and added to it. The final solution was transferred to a glass tube with two exits and was purged with nitrogen gas for 5 minutes. The copolymerization reaction was conducted for 24 hours. Hydrogels samples were washed with water, cut and dried in the oven at $60^{\circ} \mathrm{C}$ for 24 hours.

\subsection{Swelling Studies}

Hydrogels dried samples were weighted and added to $100 \mathrm{ml}$ of distilled water at room temperature. After 24 hours, samples were weighed again. Swelling was calculated by Equation (1).

$$
S=\left(\frac{W_{s}-W_{d}}{W_{d}}\right)
$$

where $W_{s}$ is the swollen weigh and $W_{d}$, the dried weigh of the samples.

\subsection{Dynamic Light Scattering Experiments}

With the purpose of studying the dynamic correlation length for the synthesized systems, dynamic light scattering measurements were carried out at $25^{\circ} \mathrm{C}$, after a reaction time of 24 hours, using a goniometer equipped with a photon detector (EG \& G SPCM) and a He-Ne laser ( $60 \mathrm{~mW}$ wavelength of $632.8 \mathrm{~nm}$ ). The scattering angle was fixed at $90^{\circ}$. 
Syntheses were made for both crosslinkers using the same procedure as before but gelification occurred in Eppendorf tubes. After 24 hours, hydrogels samples were washed with water, transferred to a sample holder with $20 \mathrm{~mL}$ of water and were left resting 24 hours to swell.

\section{Results and Discussion}

By fixing the amount of crosslinker and NMA content and by varying the total monomer concentration hydrogels phase transitions were found. Then new syntheses were made fixing total monomer concentration and increasing the amount of ionic monomer to evaluate their swelling behavior near phase transition and deviating from it, for both crosslinkers. Dynamic Light Scattering experiments were also performed for hydrogels near their phase transition to measure the dynamic correlation length, which is associated to spatial gel inhomogeneities. The results are presented and discussed in this section.

\subsection{Phase Transition Experiments}

First, hydrogels were synthesized varying $\mathrm{X}$ values and maintaining $1 \% \mathrm{Y}$ and $30 \% \mathrm{Z}$ fixed. The first $\mathrm{X}$ values were chosen based on previous work [16]. Table 1 presents synthesis parameters for MBA and EGMA.

For MBA, gelations were successful for all compositions, but there was no reproducibility for 5:1:30. Syntheses with EGMA were successful until 15\% of total monomers but the composition 10:1:30 formed a polymeric solution. New experiments were made between $14 \%$ and $11 \%$ of total monomers and it was possible to obtain consistent hydrogels until $12 \%$ of X. To guarantee system stability, $6 \%$ and $14 \%$ of total monomers were chosen for MBA and for EGMA new syntheses, respectively. Comparing the two limits achieved with different crosslinkers, we can see that MBA is more effective than EGMA to form consistent hydrogels with less total monomers.

\subsection{Swelling Behavior for Both Crosslinkers}

Once the phase transitions for both systems were found, new experiments were made maintaining $\mathrm{X}$ and $\mathrm{Y}$ constant and varying $\mathrm{Z}$ from $0 \%$ to $35 \%$, as is presented in Table 2 .

Swelling measurements were conducted for all samples and the results are presented at Figure 1.

As it can be seen, NMA concentration affects hydrogels swelling behavior. With no NMA content, swelling is very low but increases with $\mathrm{Z}$ up to $20 \%$. This result is quite expected, since ionic monomers promote more sites for hydrogen bonds. Then, swelling decreases in the range of $25 \%-30 \%$ of $Z$, increases again from $30 \%$ to $35 \%$ and decreases again, oscillating around $285 \mathrm{~g} / \mathrm{g}$ for MBA and $325 \mathrm{~g} / \mathrm{g}$ for EGMA crosslinker.

Both crosslinkers presented great swelling results, although EGMA hydrogels expands more in water compared to MBA ones. It can also be seen that changes in swelling behavior are more abrupt for EGMA than to MBA hydrogels.

New experiments were made to test hydrogels behavior deviating from phase transition. The chosen compositions are listed at Table 3.

Figure 2 and Figure 3 presents swelling behavior for these hydrogels.

Until $10 \%$ of NMA, all hydrogels made with MBA crosslinker behave similarly, but above this content, the $6: 1: Z$ curve shows a better swelling behavior compared to others. It seems that increasing total monomer concentration lead to hydrogels with larger and more entangled chains, so that they cannot swell as much as before.

The behavior for hydrogels with EGMA is similar to MBA ones until $20 \%$ of NMA. After that point, while the $18: 1: \mathrm{Z}$ curve stabilizes around 200 to $250 \mathrm{~g} / \mathrm{g}$ of expansion, the $16: 1: \mathrm{Z}$ and $20: 1: \mathrm{Z}$ show increasing swelling until $30 \%$ of NMA with a maximum peak for $16: 1: Z$ of $377 \mathrm{~g} / \mathrm{g}$.

Comparing Figure 2 and Figure 3, it is possible to infer that EGMA crosslinker provides hydrogels with greater swelling behavior than the ones with MBA. For instance, the maximum expansion with MBA, for 6:1:35, was $397 \mathrm{~g} / \mathrm{g}$, while with EGMA, for 16:1:35, was $490 \mathrm{~g} / \mathrm{g}$, almost one hundred times bigger.

Comparing to literature, Raju et al. [5] obtained maximum swelling of $191 \mathrm{~g} / \mathrm{g}$ and Karadag et al. [10], $88 \mathrm{~g} / \mathrm{g}$, for hydrogels based on acrylamide and sodium methacrylate, using EGMA as crosslinker. These so different results for similar systems show that chain length, entanglement and network strength and ultimately, swelling behavior are greatly affected by total monomer, ionic monomer and crosslinker concentration. 
Table 1. Phase transition experiments.

\begin{tabular}{cccccc}
\hline & Syntheses using MBA & \multicolumn{3}{c}{ Syntheses using EGMA } \\
\hline $\mathrm{X}$ & $\mathrm{Y}$ & $\mathrm{Z}$ & $\mathrm{X}$ & $\mathrm{Y}$ & $\mathrm{Z}$ \\
\hline 10 & 1 & 30 & 50 & 1 & 30 \\
9 & 1 & 30 & 45 & 1 & 30 \\
8 & 1 & 30 & 40 & 1 & 30 \\
7 & 1 & 30 & 35 & 1 & 30 \\
6 & 1 & 30 & 30 & 1 & 30 \\
5 & 1 & 30 & 25 & 1 & 30 \\
& & & 20 & 1 & 30 \\
& & & 15 & 1 & 30 \\
\end{tabular}

Table 2. Experiments with increasing NMA content.

\begin{tabular}{cccccc}
\hline & Syntheses using MBA & \multicolumn{3}{c}{ Syntheses using EGMA } \\
\hline $\mathrm{X}$ & $\mathrm{Y}$ & $\mathrm{Z}$ & $\mathrm{X}$ & $\mathrm{Y}$ & $\mathrm{Z}$ \\
\hline 6 & 1 & 0 & 14 & 1 & 0 \\
6 & 1 & 10 & 14 & 1 & 10 \\
6 & 1 & 20 & 14 & 1 & 20 \\
6 & 1 & 25 & 14 & 1 & 25 \\
6 & 1 & 30 & 14 & 1 & 30 \\
6 & 1 & 35 & 14 & 1 & 35 \\
6 & 1 & 40 & 14 & 1 & 40 \\
6 & 1 & 45 & 14 & 1 & 45 \\
6 & 1 & 50 & 14 & 1 & 50 \\
\hline
\end{tabular}

Table 3. Experiments with NMA and total monomers variation.

\begin{tabular}{ccccccc}
\hline & 1 & 2 & 3 & 4 & 5 & 6 \\
\hline \multirow{3}{*}{ MBA } & $8: 1: 0$ & $8: 1: 10$ & $8: 1: 20$ & $8: 1: 25$ & $8: 1: 30$ & $8: 1: 35$ \\
& $10: 1: 0$ & $10: 1: 10$ & $10: 1: 20$ & $10: 1: 25$ & $10: 1: 30$ & $10: 1: 35$ \\
& $12: 1: 0$ & $12: 1: 10$ & $12: 1: 20$ & $12: 1: 25$ & $12: 1: 30$ & $12: 1: 35$ \\
& $16: 1: 0$ & $16: 1: 10$ & $16: 1: 20$ & $16: 1: 25$ & $16: 1: 30$ & $16: 1: 35$ \\
EGMA & $18: 1: 0$ & $18: 1: 10$ & $18: 1: 20$ & $18: 1: 25$ & $18: 1: 30$ & $18: 1: 35$ \\
& $20: 1: 0$ & $20: 1: 10$ & $20: 1: 20$ & $20: 1: 25$ & $20: 1: 30$ & $20: 1: 35$
\end{tabular}

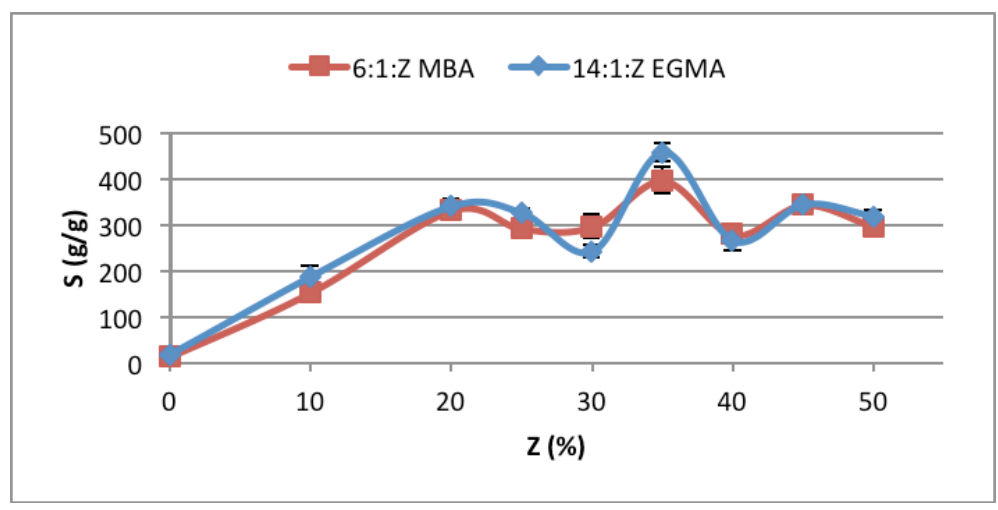

Figure 1. Swelling behavior of EGMA and MBA hydrogels with increasing NMA content. 


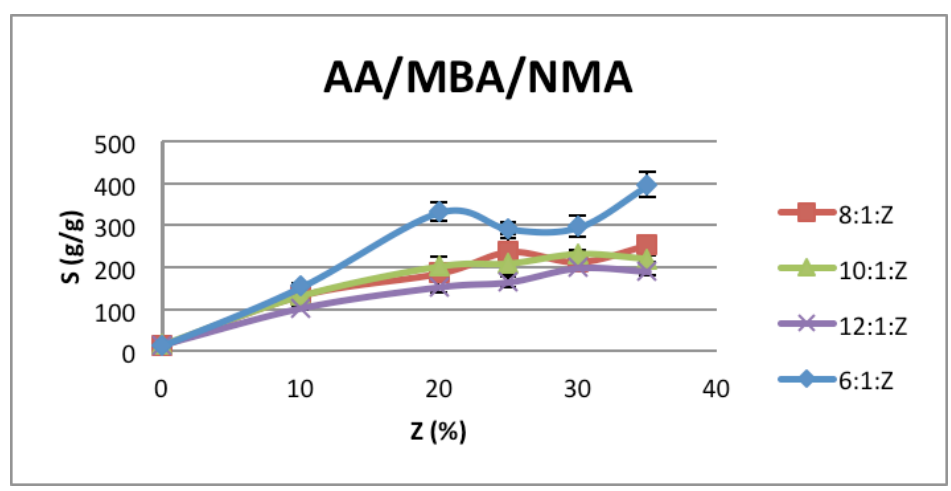

Figure 2. Comparison of swelling behavior of MBA hydrogels with increasing NMA content.

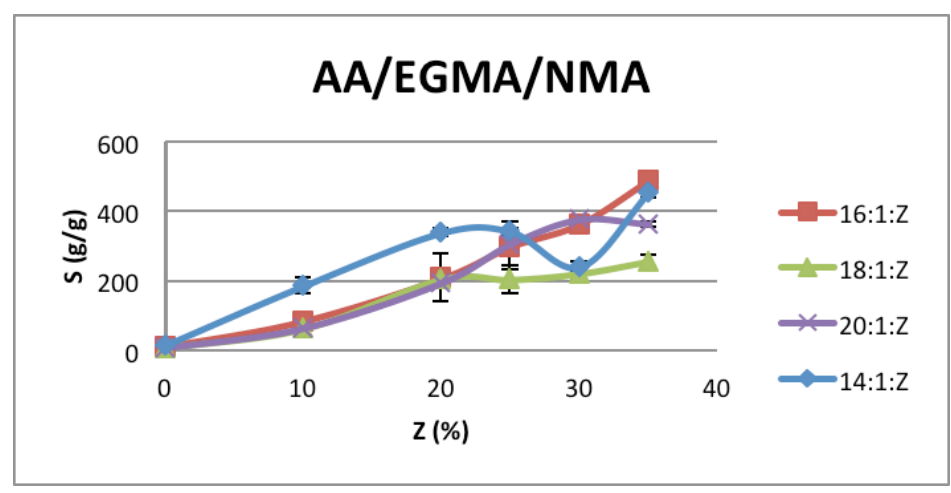

Figure 3. Comparison of swelling behavior of EGMA hydrogels with increasing NMA content.

\subsection{Dynamic Light Scattering Results}

The chosen compositions for dynamic light scattering experiments were the phase transition ones: $6: 1: \mathrm{Z}$ for MBA and 14:1:Z for EGMA, varying NMA content from $0 \%$ to $45 \%$.

Results are presented at Figure 4, where the upper graphic represents scattering intensity and the lower one, dynamic correlation length versus NMA content. MBA hydrogels samples were tested two times within a week and are represented by red circles and blue crosses. Black stars represent EGMA hydrogels samples. As it can be seen, for both crosslinkers, the dynamic correlation length $(\xi)$ decreases with increasing NMA content. As it represents an average distance between entanglements, results show that the ionic monomer provides higher interaction and entanglement between network chains, contributing to diminish spatial inhomogeneities in hydrogels.

\section{Conclusion}

Hydrogels based on AA and NMA using MBA or EGMA as crosslinkers were developed and their phase transition was found. New syntheses were made with increasing amount of ionic monomer and their swelling behavior was evaluated. Both crosslinkers presented great swelling results, although EGMA hydrogels expands more in water compared to MBA ones. Deviating from phase transition, i.e. increasing total monomer concentration, leaded to less expansive hydrogels, probably due to larger and more entangled chains. Dynamic Light Scattering experiments were performed for hydrogels near their phase transition and it was seen that dynamic correlation length decreased with increasing sodium methacrylate content.

\section{Acknowledgements}

The authors thank the Polymer Science and Technology Laboratory of Federal University of Minas Gerais for 


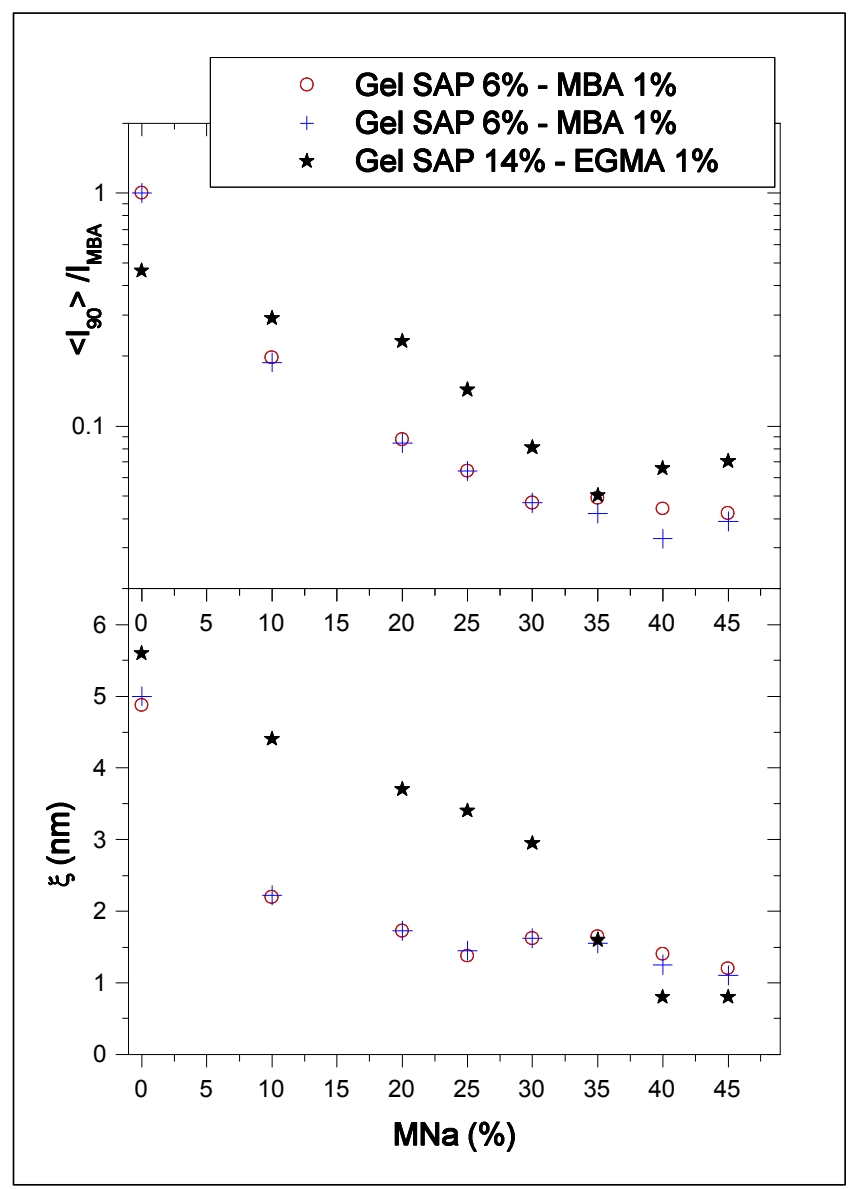

Figure 4. Dynamic light scattering results.

all support to this research.

\section{References}

[1] Buchholz, F.L. and Graham, A.T. (Eds.) (1998) Modern Superabsorbent Polymer Technology. 1st Edition, WileyVCH, New York.

[2] Zohuriaan-Mehr, M.J., Omidian, H., Doroudiani, S. and Kabiri, K. (2010) Advances in Non-Hygienic Applications of Superabsorbent Hydrogel Materials. Journal of Materials Science, 45, 5711-5735. http://dx.doi.org/10.1007/s10853-010-4780-1

[3] Raju, K.M., Mohan, Y.M. and Raju, M.P. (2002) Synthesis and Water Absorbency of Crosslinked Superabsorbent Polymers. Journal of Applied Polymer Science, 85, 1795-1801. http://dx.doi.org/10.1002/app.10731

[4] Raju, K.M., Mohan, Y.M., Murthy, S.P.K., Rao, K.M. and Sreeramulu, J. (2005) Swelling Behavior and Diffusion Studies of High-Water-Retaining Acrylamide/Potassium Methacrylate Hydrogels. Journal of Applied Polymer Science, 96, 1153-1164. http://dx.doi.org/10.1002/app.21565

[5] Raju, K.M., Mohan, Y.M. and Murthy, S.K. (2005) Synthesis, Characterization and Effect of Reaction Parameters on Swelling Properties of Acrylamide-Sodium Methacrylate Superabsorbent Copolymers. Reactive and Functional Polymers, 63, 11-26. http://dx.doi.org/10.1016/j.reactfunctpolym.2005.01.005

[6] Raju, K.M., Mohan, Y.M., P. Murthy, S.P.K. and Sreedhar, B. (2006) Swelling and Thermal Characteristics of pH Sensitive Crosslinked Poly(Acrylamide-co-Calcium Methacrylate) Superabsorbent Copolymers. Journal of Applied Polymer Science, 102, 1-12. http://dx.doi.org/10.1002/app.23562

[7] Karadag, E., Saraydin, D. Çaldiran, Y. and Güven, O. (2000) Swelling Studies of Copolymeric Acrylamide/crotonic acid Hydrogels as Carriers for Agricultural Uses. Polymers Advanced Technologies, 11, 59-68. http://dx.doi.org/10.1002/(SICI)1099-1581(200002)11:2<59::AID-PAT937>3.0.CO;2-Z 
[8] Karadag, E. and Saraydin, D. (2002) Swelling of Superabsorbent Acrylamide/Sodium Acrylate Hydrogels Prepared Using Multifunctional Crosslinkers. Turkish Journal of Chemistry, 26, 863-875.

[9] Karadag, E., Saraydin, D. and Baris Üzüm, O. (2005) Water Uptake in Chemically Crosslinked Poly(Acrylamide-coCrotonic Acid) Hydrogels. Materials \& Design, 26, 265-270. http://dx.doi.org/10.1016/j.matdes.2004.07.014

[10] Karadag, E., Kiristi, T., Kundakci, S. and Baris Üzüm, O. (2010) Investigation of Sorption/swelling Characteristics of Chemically Crosslinked AAm/SMA Hydrogels as Biopotential Sorbent. Journal of Applied Polymer Science, 117, 17871797. http://dx.doi.org/10.1002/app.32125

[11] Yildiz, B., Isik, B. and Kis, M. (2002) Thermoresponsive Poly(N-Isopropylacrylamide-co-Acrylamide-co-2-Hydroxyethyl Methacrylate) Hydrogels. Reactive \& Functional Polymers, 52, 3-10. http://dx.doi.org/10.1016/S1381-5148(02)00035-4

[12] Bakass, M., Bahaj, H., Benaddi, R. and Bayane, C. (2009) Swelling of Superabsorbents Polymers in an Aqueous Medium. Journal of Applied Polymer Science, 115, 2479-2484. http://dx.doi.org/10.1002/app.31030

[13] Okay, O. and Sariisik, S.B. (2000) Swelling Behavior of Poly(Acrylamide-co-Sodium Acrylate) Hydrogels in Aqueous Salt Solutions: Theory versus Experiments. European Polymer Journal, 36, 393-399. http://dx.doi.org/10.1016/S0014-3057(99)00058-0

[14] Wan, T., Wang, L., Yao, J., Ma, X., Yin, Q. and Zang, T. (2008) Saline Solution Absorbency and Structure Study of Poly(AA-AM) Water Superabsorbent by Inverse Microemulsion Polymarization. Polymer Bulletin, 60, 431-440. http://dx.doi.org/10.1007/s00289-007-0875-5

[15] Okay, O., Kuru, E. A. and Orakdogen, N. (2007) Preparation of Homogeneous Polyacrylamide Hydrogels by FreeRadical Crosslinking Copolymerization. European Polymer Journal, 43, 2913-2921. http://dx.doi.org/10.1016/j.eurpolymj.2007.04.019

[16] Leite, S.A.F. (2009) Contribuição ao Estudo de Polímeros Superabsorventes: Síntese e Caracterização de Hidrogéis a partir da Copolimerização de Acrilamida com Metacrilatos. M.Sc. Thesis, Universidade Federal de Minas Gerais, Belo Horizonte. 
Scientific Research Publishing (SCIRP) is one of the largest Open Access journal publishers. It is currently publishing more than 200 open access, online, peer-reviewed journals covering a wide range of academic disciplines. SCIRP serves the worldwide academic communities and contributes to the progress and application of science with its publication.

Other selected journals from SCIRP are listed as below. Submit your manuscript to us via either submit@scirp.org or Online Submission Portal.
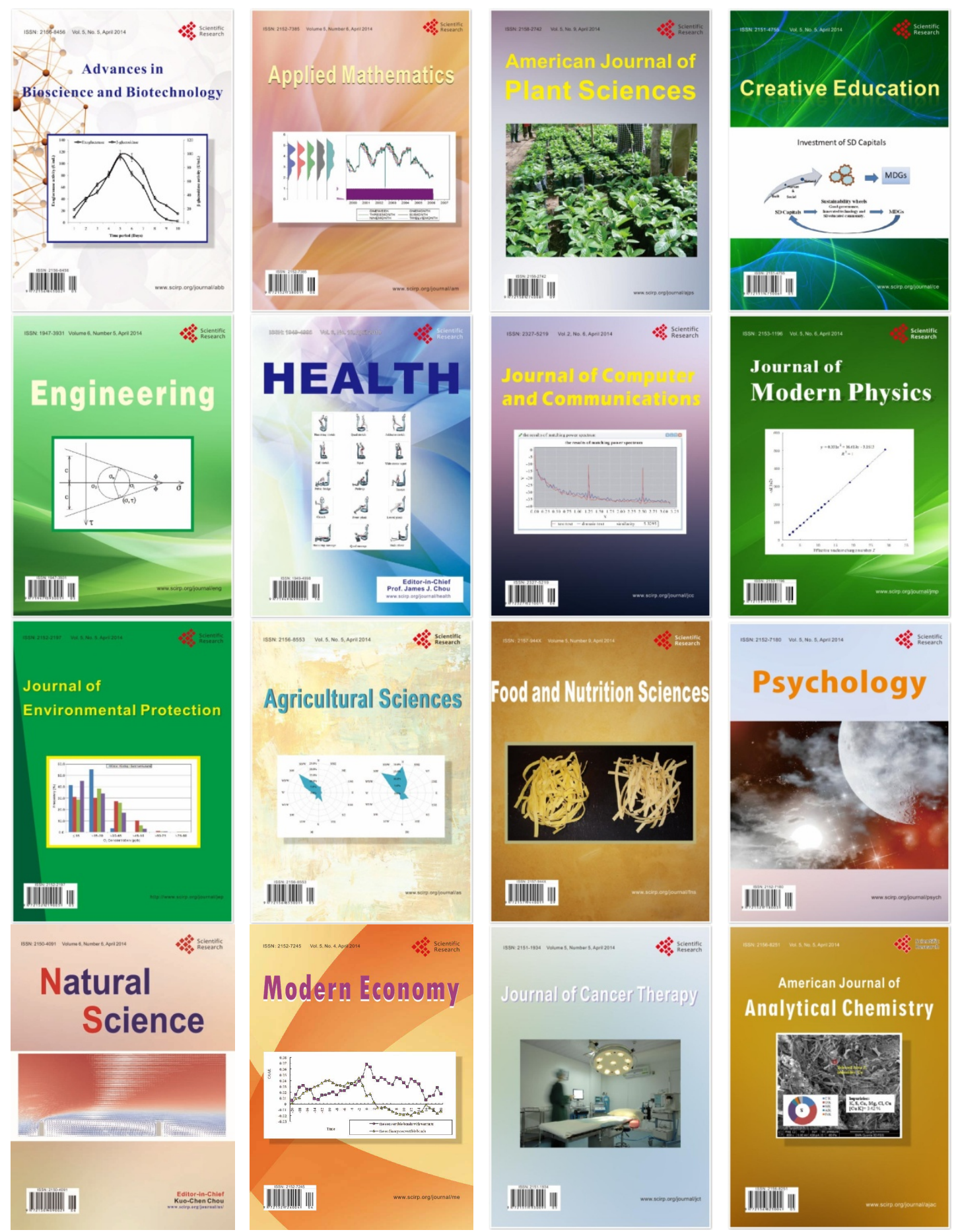